\title{
PACKAGING OF LARGE LATERAL DEFLECTION MEMS USING A COMBINATION OF FUSION BONDING AND EPITAXIAL REACTOR SEALING
}

\author{
M. W. Messana* , A. B. Graham, S. Yoneoka, R. T. Howe, and T. W. Kenny \\ Stanford University, Stanford, California, USA
}

\begin{abstract}
The fabrication process described in this paper combines fusion bonding with a thin film sealing technique to package various MEMS devices. This combination allows large variations in trench widths $(1.5 \mu \mathrm{m}$ up to $100 \mu \mathrm{m})$, which is necessary for encapsulating structures that include combdrives or large parallel plate capacitor arrays. The design rules are flexible enough to allow many different capacitively actuated, lateral displacement MEMS devices. Structures with resonant frequencies ranging from $5 \mathrm{kHz}$ up to $1.4 \mathrm{MHz}$ have been successfully encapsulated using this process with yields greater than $80 \%$. In contrast to many other wafer bonding methods, fusion bonding allows the footprint of these fully encapsulated structures to be only slightly larger than the structure itself.
\end{abstract}

\section{INTRODUCTION}

Packaging is often one of the most important design considerations in microelectromechanical systems (MEMS). It must provide a clean and stable environment for the device to reside in as well as allow electrical connection to the outside world. Ideally, the packaging would be low-cost and robust enough to withstand harsh post-processing (such as wafer dicing and wire bonding) while being fully integrated into a final system.

Traditional packaging techniques involve assembling each device into its final wafer package after singulation. This is expensive because each complex package is created individually. Additionally, if the MEMS structures are released prior to singulation, there will likely be a significant reduction in yield. Wafer level packaging techniques attempt to address these issues by integrating the device packaging into the overall fabrication process. Najafi provides a good overview of the various wafer level packaging methods commonly utilized in MEMS [1].

Anodic and eutectic bonding are commonly used to encapsulate MEMS devices at the wafer level. Typically, a wafer with aligned pockets is bonded to a wafer with released devices. While sometimes able to produce a very hermetic package, these bonding techniques typically require large bond areas which increases cost due to a reduction in the number of devices that can be made on a single wafer. Additionally, making electrical contact to the encapsulated devices often requires lateral traces which further increase the package footprint.

For thin film encapsulation, a sacrificial material is usually deposited over the MEMS structures, followed by the deposition of the encapsulation layer material. Upon removal of the sacrificial material, the released devices are re-sealed in their cavity. Depending on the materials used, this can result in a very hermetic package with a much smaller overall footprint than the wafer bonding techniques described above. However, if thicker device layers are desired, which provide improved performance in capacitively actuated and sensed devices, the design rules for thin film encapsulation techniques are typically very limiting. The sacrificial material must either bridge over the trenches defining the structures or be thick enough to fill them entirely. Both methods have been accomplished, however the design rules were either very restricting or the fabrication process impractical.

Candler et al. encapsulated piezoresistive and capacitive structures by releasing devices using hydrofluoric acid vapor and then sealing the etch access holes using low pressure chemical vapor deposition (LPCVD) silicon dioxide [2]. Later iterations of this process improved the stability performance by sealing the etch access holes in an epitaxial silicon reactor, demonstrating excellent long term stability [3]. Both of these methods relied on the sacrificial material bridging over trenches approximately $2 \mu \mathrm{m}$ wide, thus limiting designs to these narrow gaps. This precludes the incorporation of such traditional structures as combdrives. Ayanoor-Vitikkate et al. attempted to address this problem by thermally oxidizing sacrificial beams in the device layer to create a thick sacrificial material, but working devices were never demonstrated [4]. Recently, Graham et al. presented a method for successfully encapsulating MEMS devices capable of $20 \mu \mathrm{m}$ of lateral deflection [5]. While both narrow and wide trench width devices were fabricated, the process relied on the deposition and partial planarization of a thick sacrificial oxide exceeding $20 \mu \mathrm{m}$ in thickness.

The process described in this work is intended to expand the design rules of the process outlined by Kim et al. to allow various trench widths while maintaining its long term stability [3]. To bridge the large trenches, a sacrificial wafer is fusion bonded to the device wafer. Fusion bonding is preferable to other types of bonding because it requires less bond area and all of the materials and processes involved with fusion bonding are CMOS compatible. This bonded wafer is subsequently thinned and small vent holes are etched for releasing the buried devices. The resulting cavity is then resealed with epitaxially grown silicon to fully encapsulate the released devices. Figure 1 depicts a released and sealed device using this process.

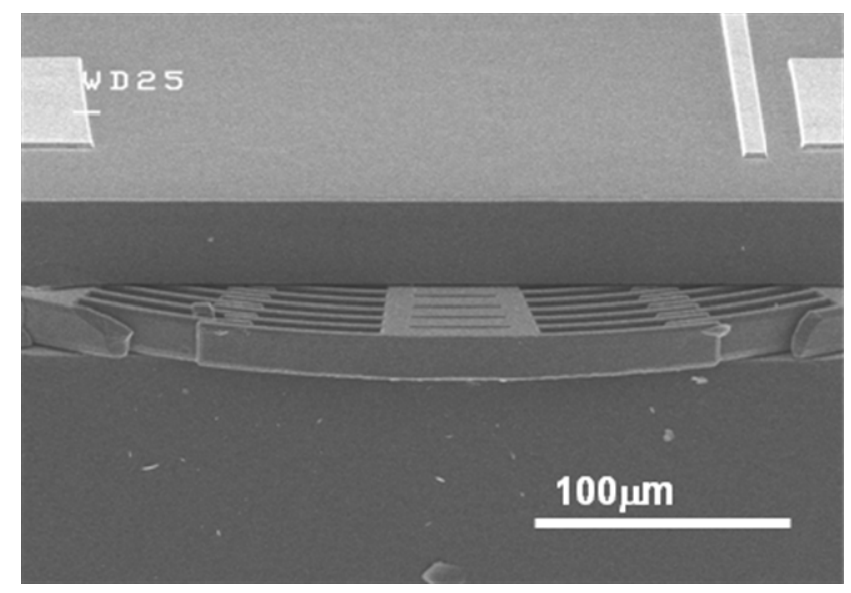

Figure 1: SEM image showing a resonating fan structure that is driven by combdrives. The metal electrical contacts can be seen on top of the encapsulation wafer.

\section{FABRICATION PROCESS}

The process, shown in Figure 2, begins with a fusion bonded SOI wafer having a $20 \mu \mathrm{m}$ thick device layer and a $2 \mu \mathrm{m}$ thick buried oxide layer. Devices are patterned in photoresist and etched into the device layer using deep reactive ion etching (DRIE), as shown in Figure 2(a). The etch recipe used in this step was tuned to minimize feature blowout, eliminate grass formation, and 
(a)
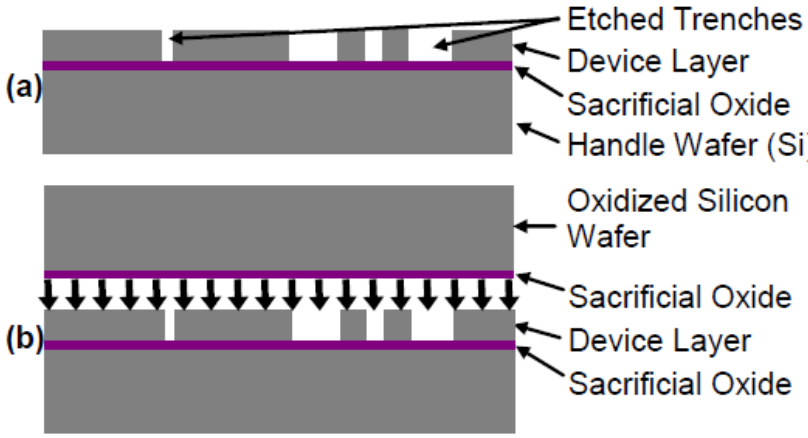

(c)

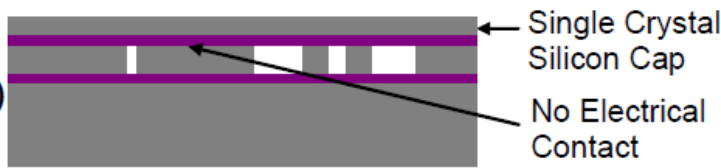

(d)

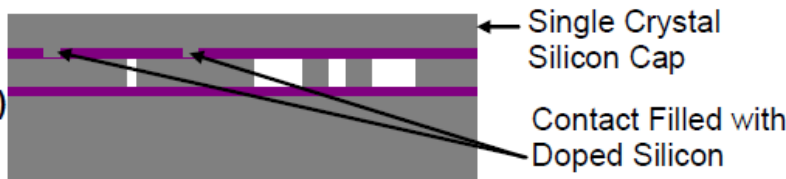

(e)

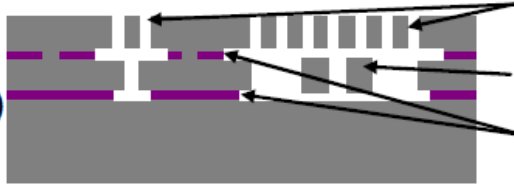

Vent Holes for

Device Release

Released

Structure

HF Vapor Etch

Fronts
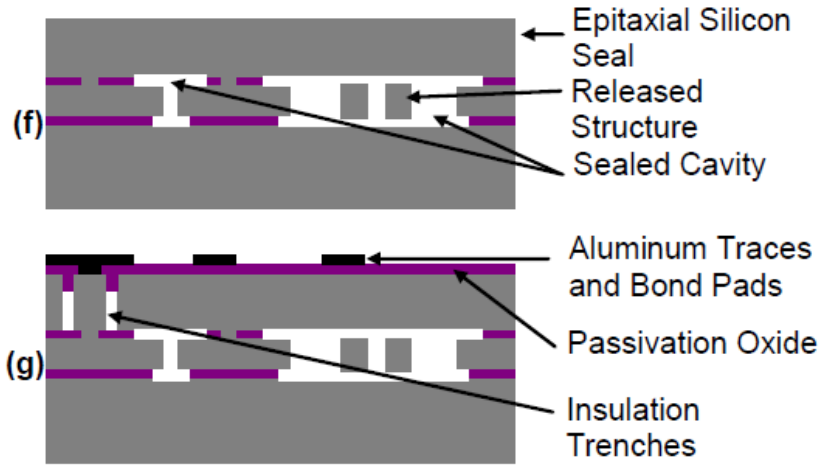

Figure 2: Schematic cross section of the encapsulation process. (a) Devices are defined in SOI wafer with a DRIE etch. (b) An oxidized silicon wafer is fusion bonded to the patterned SOI wafer. (c) The cap wafer is ground and polished to a specified thickness. (d) Electrical contact is made by etching holes and refilling with silicon. (e) Vents are etched in the cap layer and HF vapor removes $\mathrm{SiO}_{2}$ in the cavity. ( $f$ ) The cavity is sealed with silicon in an epitaxial reactor. (g) Electrical isolation trenches are etched, insulation oxide is deposited and etched, and metal is sputtered and etched.

minimize footing at the buried oxide interface. The sidewall polymer is removed using a short $\mathrm{O}_{2}$ plasma etch and the resist is stripped in a sulfuric acid solution.

In preparation for wafer bonding, both this patterned SOI and a blank silicon wafer with $2 \mu \mathrm{m}$ of thermally grown oxide receive a standard RCA-2 clean. In addition to this clean, a final clean of the wafers in a 5:1:1 mixture of $\mathrm{H}_{2} \mathrm{O}: \mathrm{NH}_{4} \mathrm{OH}: \mathrm{H}_{2} \mathrm{O}_{2}$ at $70^{\circ} \mathrm{C}$ for 20 minutes is performed in an effort to increase the bond strength [6].
The two wafers are then bonded together using a Karl Suss fusion bonding tool at a pressure less than $1 \mathrm{mTorr}$. Since the encapsulation wafer is unpatterned, a flat alignment between the two wafers is sufficient for this process. To increase the bond strength as described by Plößl et al. [7], the bonded wafer stack is annealed at $1100^{\circ} \mathrm{C}$ for 4 hours. Vacuum bonding is used because the etched features create various sized cavities that may degrade the bond local to the devices as a result of the pressure increase during the subsequent high temperature steps. As shown in Figure 2 (c), the bonded wafer is then thinned to approximately $15 \mu \mathrm{m}$ via a combination of grinding and polishing. After thinning, the wafer stack is annealed for an additional 20 hours at $1100^{\circ} \mathrm{C}$ to further improve bond strength. This overall process of bonding followed by grinding and polishing is very similar to the process used to make fusion bonded SOI wafers [8].

Contact vias are then patterned on the thinned wafer and etched to the oxide on top of the electrodes using DRIE. The oxide is cleared from the bottom of the vias using HF vapor. The vias are then filled with highly doped silicon using a process similar to filling trench capacitors in DRAM [9]. For this process, the filling of these trenches was performed in an epitaxial silicon reactor to maintain single crystal silicon across the bulk of the encapsulation wafer.

Thin vents $(0.7 \mu \mathrm{m}$ wide $)$ are then patterned and etched in the encapsulation layer using a DRIE recipe optimized for high aspect ratio trenches. The $\mathrm{SiO}_{2}$ on the top and bottom of the device is removed using a timed HF vapor etch. An IR microscope is used to confirm that the devices are fully released as shown in Figure 3.

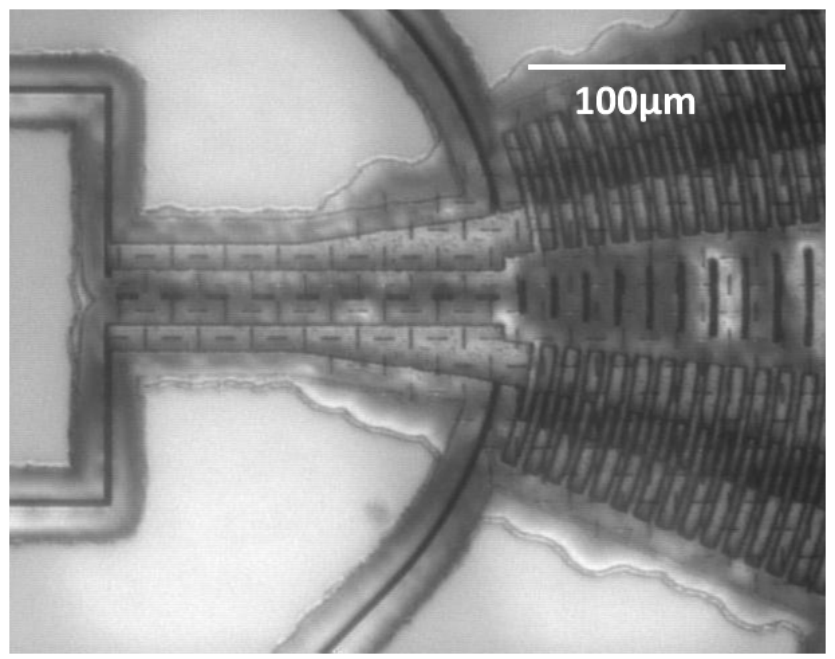

Figure 3: IR image of a MEMS device encapsulated in this process. The cross hatches over the structure are the vents that allow HF vapor to remove the $\mathrm{SiO}_{2}$ off the top and bottom of the device. Vapor etch fronts can be seen in the image and, with the exception of where the vents are located, the top and bottom vapor etch fronts are nearly on top of each other.

Immediately following the release, the wafers are placed in an epitaxial silicon reactor. The wafers are first baked at $1130^{\circ} \mathrm{C}$ for 5 minutes to remove any native oxide that might have formed on the wafer surface after vapor etching [10]. This step also serves to smooth out the scallop features left by the DRIE. The vents are then sealed with silicon at high temperature $\left(925^{\circ} \mathrm{C}\right)$ and low pressure (20Torr) using a recipe that selectively deposits on silicon over silicon dioxide. This selectivity prevents electrical shorting between the encapsulation layer and the device layer across the 


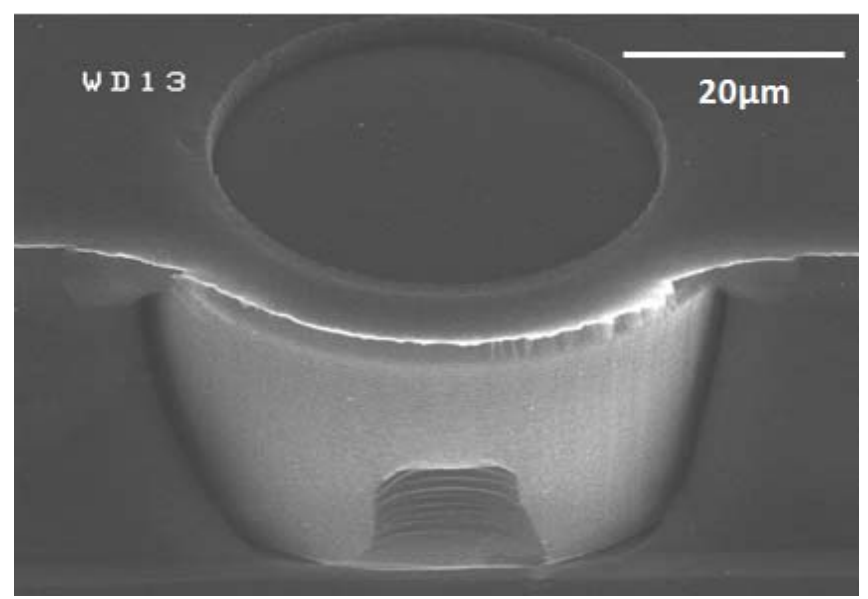

Figure 4: SEM image showing a vertical electrical connection. The circle on top is a via etched through the passivation oxide which has been covered with metal to contact the silicon plug below. The cylindrical plug is the electrical contact through the cap to the device layer below.

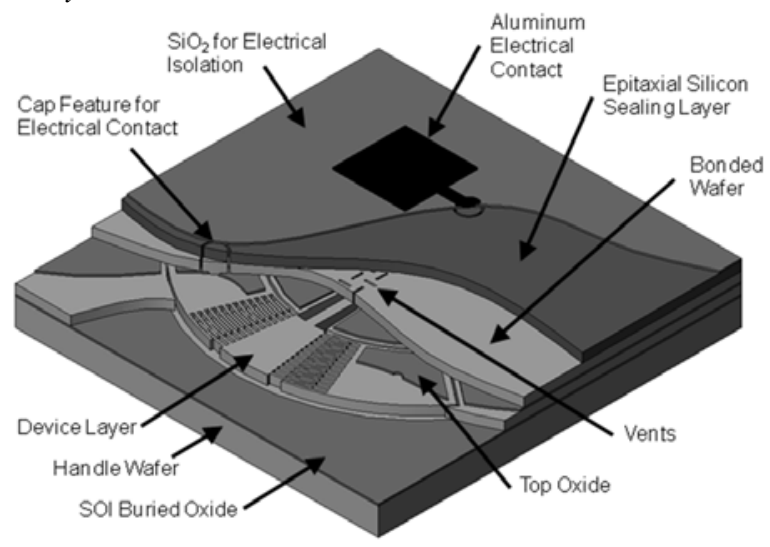

Figure 5: Isometric view of all the layers of the final packaged device.

sacrificial oxide. With hydrogen as a carrier gas, the reaction gases, $\mathrm{H}_{2} \mathrm{SiCl}_{2}, \mathrm{PH}_{3}$, and $\mathrm{HCl}$, represent less than $4 \%$ of the composition of gases in the chamber. This sealing process results in a cavity free of native oxide that contains primarily hydrogen as a residual gas. To increase the strength of the cap layer, approximately $6 \mu \mathrm{m}$ of additional silicon is added to the silicon needed to seal the vents. To improve subsequent lithography steps, the wafers are polished using a CMP, removing approximately $1 \mu \mathrm{m}$ of silicon in the process. A schematic cross section of the wafer stack at this step can be seen in Figure 2(f).

The remaining steps are needed to make isolated electrical contact to the devices and the various electrodes needed to actuate the device. Isolation trenches are patterned around the contact vias and etched to the top oxide using DRIE. Approximately $3 \mu \mathrm{m}$ of LPCVD $\mathrm{SiO}_{2}$ is deposited on the wafer to cover over the isolation trenches. Contact vias are patterned and plasma etched into this oxide to allow metal contact to the cap layer.

To create the wire traces and bond pads, aluminum is sputtered on the top surface of the wafer. This metal layer is plasma etched to complete the process, as shown in Figure $2(\mathrm{~g})$. An SEM showing the complete vertical interconnect is shown in Figure 4. An isometric view of the final wafer stack can be seen in Figure 5.

\section{RESULTS AND DISCUSSION}

Using the process described in this paper, numerous working devices were fabricated and successfully encapsulated. These devices include double ended tuning fork (DETF) resonators with resonant frequencies ranging from $190 \mathrm{kHz}$ to $1.4 \mathrm{MHz}$ and combdriven, fan shaped resonators with resonant frequencies ranging from $50 \mathrm{kHz}$ to $150 \mathrm{kHz}$. In addition to these, many other resonant structures, inertial sensors, and structures for fundamental studies of such things as fatigue and friction were included, but have yet to be tested.

As was demonstrated by Candler et al., the pressure inside the cavity can be reduced by elevated temperature $\left(400^{\circ} \mathrm{C}\right)$ annealing in a nitrogen environment [11]. While this is not necessary or even desirable for all MEMS devices, a decrease in pressure leads to an increase in the quality factor of many types of resonators. This trend is shown in Figure 6 for two different designs of tuning fork resonators. Figure 7 shows the change in the frequency response plot for one such resonator for three different times throughout the

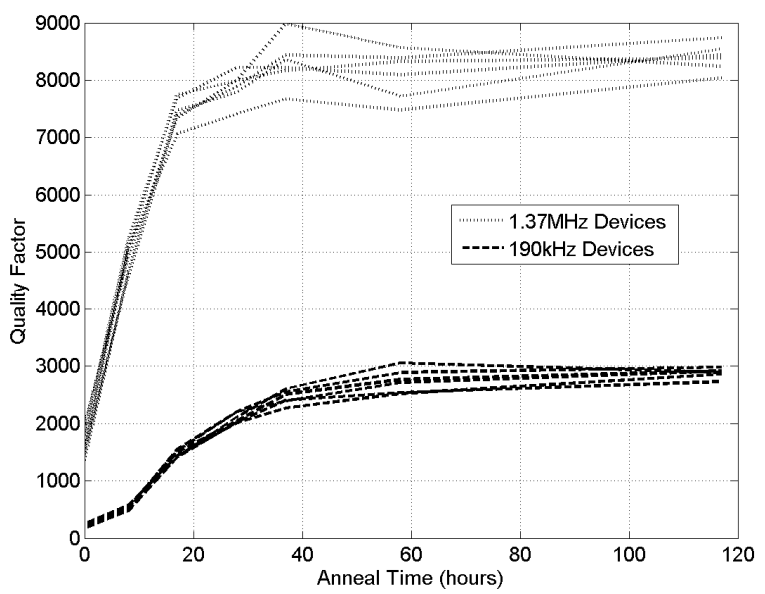

Figure 6: This plot shows resonator quality factor, Q, increasing during a $400{ }^{\circ} \mathrm{C}$ anneal in a nitrogen ambient. Increasing quality factor corresponds to a decrease in the cavity pressure. Data is shown for multiple DETF resonators at $1.37 \mathrm{MHz}$ and $190 \mathrm{kHz}$.
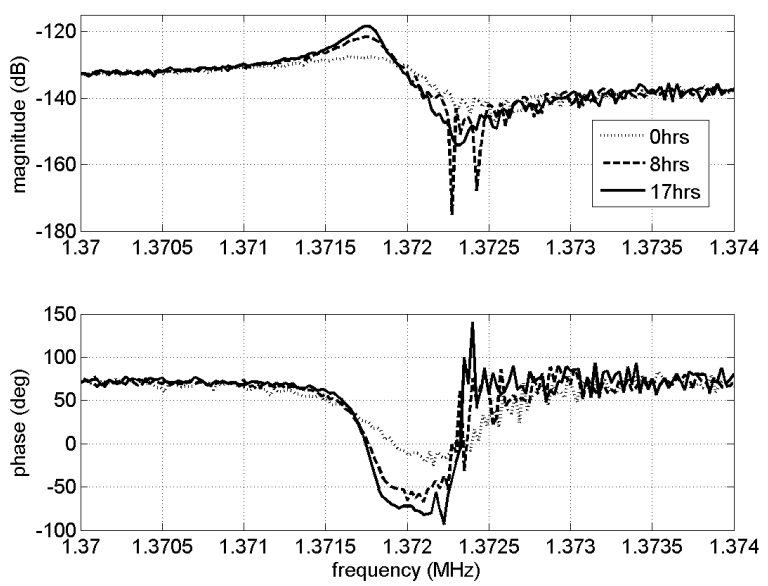

Figure 7: This plot shows example frequency response plots for a $1.37 \mathrm{MHz}$ double ended tuning fork resonator. The multiple lines correspond to different times during a $400^{\circ} \mathrm{C}$ anneal. Signal quality is improving with increased anneal time. 
anneal. The amount of time required for the resonators' quality factor to reach a maximum is comparable with that previously reported for packages shown to be quite hermetic $[3,5]$.

Table 1 gives a summary of the yield for several different resonating structures tested on a single wafer for 20 instances of each design. The yield being lower than $100 \%$ can be attributed to slight process variations such as particles on the wafers during bonding, various etch or lithography problems, and possible shorting resulting from an epitaxial reactor seal recipe that was not perfectly selective in its deposition on silicon over silicon dioxide.

Table 1: Summary of yield for various devices on a single wafer.

\begin{tabular}{|l|c|}
\hline Device & Yield \\
\hline Single anchored 1.38MHz DETF & $75 \%$ \\
\hline Double anchored 1.4MHz DETF & $65 \%$ \\
\hline Single anchored 190kHz DETF & $100 \%$ \\
\hline $100 \mathrm{kHz}$ Comb Drive Resonator & $90 \%$ \\
\hline
\end{tabular}

\section{CONCLUSION}

This new fabrication process for the wafer level packaging of MEMS devices combines two existing encapsulation techniques: fusion wafer bonding and epitaxial reactor sealing. The use of fusion bonding allows smaller bond areas than most other bonding techniques while maintaining a single crystal silicon cap and CMOS compatible materials. Because of the single crystal silicon cap layer, optical transparency is improved over the use of polysilicon, making this packaging method especially suitable for encapsulating optical devices working in the infrared range. The use of epitaxial reactor sealing provides a sealed environment free of native oxide and maintains the single crystal silicon cap layer. The combination of these two techniques has allowed the encapsulation of devices capable of large lateral deflections while not sacrificing the ability to include the smaller gaps desired for many electrostatic devices. Additionally the overall package footprint needs only to be slightly larger than the device itself.

A variety of structures were included in this process, with resonant devices having frequencies ranging from $5 \mathrm{kHz}$ to 1.4MHz. In addition, yield was quite high for a process developed and carried out in a research-oriented academic fabrication facility. Future work will focus on evaluating the hermeticity of the package by monitoring long term frequency stability and on characterizing the numerous other devices fabricated in this process.

\section{ACKNOLEDGEMENTS}

This work was performed under the Center on Interfacial Engineering for Microelectromechanical Systems, funded by DARPA grant HROO11-06-0049 and managed by Dr. D. L. Polla. Additional support was provided by the National Nanofabrication Users Network facilities funded by the National Science Foundation under award ECS-9731294. The authors would also like to thank Gary Yama, Mario Kupnik, and J Provine for helpful discussions. Travel support was provided by the Transducers Research Foundation for M. W. Messana.

\section{REFERENCES}

[1] K. Najafi, "Micropackaging technologies for integrated microsystems: Applications to MEMS and MOEMS," Proc. SPIE - Micromachining and Microfabrication Process Technology VIII, pp. 1-19, 2003.

[2] R.N. Candler, W.-T. Park, H. Li, G. Yama, A. Partridge, M. Lutz, and T.W. Kenny, "Single wafer encapsulation of MEMS devices," IEEE Trans. Adv. Packag., vol. 26, no. 3, pp. 227-232, 2003.

[3] B. Kim, R.N. Candler, R. Melamud, M.W. Hopcroft, S. Yoneoka, H.K. Lee, M. Agarwal, S. Chandorkar, G. Yama, and T.W. Kenny, "Hermeticity and diffusion investigation in polysilicon film encapsulation for microelectromechanical systems," Journal of Applied Physics, vol. 105, 0135142009.

[4] V. Ayanoor-Vitikkate, K.-L. Chen, W.-T. Park, and T. W. Kenny, "Development of wafer scale encapsulation process for large displacement piezoresistive MEMS devices," Sens. Actuators A, Phys., vol. 156, pp. 275-283, 2009.

[5] A.B. Graham, M.W. Messana, P.G. Hartwell, J. Provine, S. Yoneoka, R. Melamud, B. Kim, R.T. Howe, and T.W. Kenny, "A Method for wafer scale encapsulation of large lateral deflection MEMS devices," Journal of Microelectromechanical Systems, vol.19, no.1, pp.28-37, Feb. 2010.

[6] Y.-L.Chao, Q.-Y. Tong, T.-H. Lee, M. Reiche, R. Scholz, J.C.S. Woo, and U. Gösele, "Ammonium hydroxide effect on low-temperature wafer bonding energy enhancement," Electrochemical and Solid-State Letters, vol. 8, pp. G74-G77 2005.

[7] A. Plöß1, and G. Kräuter, "Wafer direct bonding: tailoring adhesion between brittle materials," Materials Science and Engineering, vol. R25, pp. 1-88, 1999.

[8] C. Harendt, W. Appel, H.-G. Graf, B. Höfflinger, and E. Penteker, "Wafer fusion bonding and its application to silicon-on-insulator fabrication," Journal of Micromechanics and Microengineering, vol. 1, pp. 145-151, 1991.

[9] M. Taguchi, S. Ando, N. Higaki, G. Goto, T. Ema, K. Hashimoto, and T. Yabu, "Dielectrically encapsulated trench capacitor cell," Electron Devices Meeting, 1986 International, vol.32, pp. 136-139, 1986

[10] S.T. Liu, L. Chan, and J.O. Borland, "Reaction kinetics of $\mathrm{SiO}_{2} / \mathrm{Si}(100)$ interface in $\mathrm{H}_{2}$ ambient in a reduced pressure epitaxial reactor," Proc. 10th Int. Conf. Chemical Vapor Deposition. Electrochem. Soc., pp. 428-434, 1987.

[11] R.N. Candler, W.-T. Park, M. Hopcroft, B. Kim, and T.W. Kenny, "Hydrogen diffusion and pressure control of encapsulated MEMS resonators," Proc. $13^{\text {th }}$ Int. Conf. SolidState Sens. and Actuators (TRANSDUCERS '05), pp. 920923, 2005.

\section{CONTACT}

*M. Messana, tel: +1-650-723-0277; mmessana@stanford.edu 